\title{
MUERTE SÚBITA EN LOS ATLETAS: LA FRONTERA ENTRE LA VIDA Y LA MUERTE
}

\section{SUDDEN DEATH ON THE ATHLETES: THE BORDER BETWEEN LIFE AND DEATH}

\author{
Maynard da Silva ${ }^{1}$, Kenia \& Vargas, Angelo L. de S. ${ }^{2}$
}

1-Universidade do Estado do Rio de Janeiro/UERJ, Laboratório de Estudos da Cultura Social urbana/LECSU

2-Universidade Federal do Rio de Janeiro/FND - UFRJ, Laboratório de Estudos da Cultura Social urbana/LECSU

MAYNARD S.K. \& VARGAS A.L.S. Muerte súbita en los atletas: la frontera entre la vida y la muerte $\overline{\text { Mot. }}$. Hum., 11(2): 52-58, 2010.

\section{RESUMEN}

El objetivo de este artículo es comprender el significado y la importancia del riesgo para desafiar el límite entre la vida y la muerte durante la enfermedad del corazón de un atleta. Esta se expresa en los discursos de los entrevistados, sobre la cuestión: ¿quién posee la responsabilidad de la eliminación de los atletas en riesgo de enfermedades del corazón?. Metodología de tipo fenomenológico del análisis del discurso. La decisión ha generado conflictos como la angustia de asumir las consecuencias de la elección ante la importancia del deporte en sus vidas. La emoción fue la base de la reacción. Hubo división entre la decisión de continuar, detenerse y la indecisión ante el hecho. La concesión está sujeta a sí mismos debido al derecho a elegir. La decisión de expulsión proviene de los propios deportistas, expresando que la vida les pertenece a ellos y tiene el sentido de su existencia. Reflejando tomar las decisiones al respecto, mostrando la importancia del deporte e incluso aceptando el riesgo de muerte.

Palabras clave: riesgo, la muerte súbita cardiaca, muerte

\section{INTRODUCCIÓN}

Durante los últimos treinta años, hay registros en el número de muertes por causas cardíacas de los deportistas (1), entre los deportistas estan, Marc Vivien Foe (Camerún), 2003; Serginho (Sao Caetano) 2004, Antonio Puerta (Sevilla - España) en 2007 entre muchos otros, menores de 35 años (2,3). La enfermedad que es más prevalente en el $50 \%$ de las muertes es la cardiomiopatía hipertrófica $(4,5)$. Causa por la cual se presento preocupación y motivó a generar acciones por parte de los responsables en el ámbito mundial.

Estudios del Comité Olímpico Internacional en Lausana, Suiza, con base en documentos de bancos de datos internacionales de entre 1966 y 2004 indican 1.101 muertes súbitas en deportistas menores de 35 años, obteniendo un promedio de 29 atletas por año, siendo los deportes con mayor incidencia el fútbol y el baloncesto $(6,7)$.

Maron (8) desarrolló un estudio sobre muerte súbita en los atletas en los Estados Unidos de América (EE.UU.), que abarca los años 1980 y 2006 en treinta y ocho (38) deportes. Se identificaron 1.866 muertes de los atletas con enfermedad cardíaca, con una prevalencia de miocardiopatía hipertrófica. De 2005 a 2006, se encontró un promedio de sesenta y seis (66) muertes por año, de los cuales el $82 \%$ de los casos fueron durante la competición o entrenamiento. Las dificultades en la recopilación de estos datos son relativas a los años anteriores a 1994, cuando los informes sobre los casos que se produjeron no eran comunes.

Hay muchos dilemas de circunstancias emocionales, médicas, financieras y familiares relacionados con la expulsión de un atleta después de sufrir un paro cardíaco (9), y el dilema del club, que puede ser el principal responsable de la decisión de permitir o no permitir al atleta a continuar jugando, indica la necesidad de una decisión.

Esta acción crea muchos conflictos para que el atleta (10), respecto a la decisión sobre la eliminación de la práctica deportiva, la cual puede presentar problemas para asumir las consecuencias de la elección, relacionado con el estatus profesional y la situación financiera, la eliminación de placer, ya que el deporte es una prioridad debido a la importancia que tiene en su vida. 
MAYNARD S.K. \& VARGAS A.L.S. Muerte súbita en los atletas: la frontera entre la vida y la muerte Mot. Hum., 11(2): 52-58, 2010.

El deporte de alto rendimiento, bajo el supuesto de que el atleta, a pesar de la presión rigurosa, busca un alto rendimiento por plena disposición personal, como la libre elección con la que el atleta cuenta y no una obligación o carga, se valora tanto emocionalmente como afectivamente de una manera agradable, de acuerdo a los intereses y capacidades (11). Sin embargo, la profesionalización del deporte hoy en día y el control del desempeño con ayuda de la tecnología, sin interferencia del atleta, generan que la actividad esté valorada solo como la acción alcanzada por el atleta, dejando de lado la persona del atleta, excluyéndolo del desarrollo de su propio desempeño $(8,9,11,12)$. En cuanto a las circunstancias relativas a la eliminación de los atletas en riesgo de muerte por problemas del corazón, estas serían responsabilidad del club deportivo.

El concepto simplista que afirma que el deporte sea saludable esta frecuentemente apuntado a la asociación lineal entre el atleta y los productos o servicios relacionados con la salud, para buscar, mantener o superar sus límites $(9,12,13)$ que marca la tendencia de la cuantificación de resultados en detrimento de la calidad de la expresión humana de los atletas.

El deporte profesional tiene implicaciones específicas entre el trabajo y la salud, el atleta se somete a la sobrecarga de entrenamiento, la obligación de participar en las competiciones a la discreción de los directores, el período limitado de vacaciones y otros temas que están relacionados con intereses políticos y económicos de los patrocinadores, los líderes de las instituciones deportivas y la fuerza sindical $(9,13)$. El deporte profesional mueve grandes volúmenes de dinero, pero hay una diferencia entre la ganancia financiera y la producción del atleta (13).

Tema a ser discutido por los líderes del deporte es la responsabilidad en los deportes, el administrador está dado por el atributo de posición, una porción de poder que le da autoridad sobre las decisiones relativas a administrar (14), incluidas las medidas de valores para la salud de los atletas. Este dirigente asume tres funciones: la eficiencia, probidad y rendición de cuentas, junto con su filosofía y el arte de gestionar tanto sus sistemas de valores y los valores de una cultura particular. Dependiendo del perfil moral del líder puede haber o no un exceso de poder el cual puede convertirse en abusos.

En el caso de los deportes (14), la estructura de poder, tiene como base institucional el club deportivo, siendo las entidades y asociaciones deportivas, las personas jurídicas de derecho privado, sujetos al orden de los objetivos establecidos por el Estado, con administradores que tienen la responsabilidad social y civil. En lo que se refiere al abuso de poder, el silencio o la inacción de la administración mediante el abuso de poder, este hecho puede interferir en la decisión de este deporte para la seguridad física y emocional de atleta.

En lo relativo a la responsabilidad, existen dos aspectos: en primer lugar, se refiere al médico, ya que él tiene la obligación de advertir sobre el peligro para la salud del atleta, la inminencia de la muerte, si continúa la actividad y en segundo lugar, el atleta, que no siempre puede discernir la severidad de su situación, y la necesidad de detener la práctica del deporte, mediante la investigación de la enfermedad $\mathrm{y}$ someterse al riesgo y, finalmente, morir de muerte súbita en el deporte activo (15).

La institución que regula la actividad deportiva, que tiene personalidad jurídica, reglamento, resolución, así como la publicidad, permite registrar el rendimiento del deportista y compararlo con el rendimiento de los demás (13) la profundización de la participación del atleta, y si es necesaria su eliminación puede provocar intensos conflictos inherentes a la toma de decisiones.

En el caso del jugador Serginho, el personal del Instituto del Corazón, informó a él y al equipo responsable de sus afecciones cardiacas, e hizo caso omiso de la recomendación de expulsión, lo que condujo quizá a su muerte, hecho el cual cayó sobre la responsabilidad civil y ética de todos. Cuestiones que pueden estar presentes en la mayoría de las muertes de varios atletas, en referencia a un estudio victimológico centrado en la omisión, ya que el código de ética profesional establece el derecho a la vida y a la salud, lo que indica el área de responsabilidad profesional en cualquier instancia trabajo (2), sugiriendo ideas profundas sobre los dilemas del deporte, de las actividades deportivas, y la posibilidad de muerte por no cesar (9). 
MAYNARD S.K. \& VARGAS A.L.S. Muerte súbita en los atletas: la frontera entre la vida y la muerte Mot. Hum., 11(2): 52-58, 2010.

A pesar de la mediación tecnológica, la profesionalización del deporte, el atleta es la persona que va a estar ausente de sus actividades y sufrirá las consecuencias de este acto (11), debe entenderse que estará en el umbral entre la vida y la muerte, con el riesgo de cualquier desafío. La responsabilidad de estar lejos de ser cesado es en lo que se centralizan los debates.

El deporte es un reto que lleva a la confrontación, por lo que se entremezcla con los riesgos (16), a pesar de señalar la importancia de respetar las reglas, indica que el enlace entre el compromiso, la competencia y los resultados exitosos pudiendo llevar al atleta a riesgo de tratar de lograr más allá de sí mismo.

Estas opciones están dadas por la cultura del individuo en el concepto social y sentido moderno de tener control sobre las emociones y pasiones, y la responsabilidad para el futuro, con seguridad, con un riesgo calculado, la vida se considera como un bien mayor, donde la seguridad es un valor indiscutible y no negociable.

En presencia de la enfermedad cardíaca, el riesgo se traduce en la incomprensión por parte del propio atleta, encargado de los deportes, la inminencia de la muerte súbita y no sujetos a control, tiene un carácter negativo. Una asociación al deporte - riesgo - emoción trae significados a las experiencias vividas por los atletas en una perspectiva romántica de dar sentido a la vida (17).

Los riesgos están relacionados con los valores y significados personales, incluso en los deportes (18), dando lugar a una brecha entre la realidad y los peligros de la representación.

La auto-superación, vinculada a la asociación entre el deporte, la emoción y el riesgo, en perspectiva del atleta, equivale a ser un ganador en la vida, esta premisa puede ser una razón que lleva a una mayor exigencia de rendimiento físico que puede llevar constante su potencial de las marcas y los índices para ser clasificado en las competiciones (19).

El deporte se considera una manifestación del movimiento humano, como resultado de la reciprocidad de la herencia biológica y la historia social, guiados por la objetividad y el significado de la vida, que está construyendo su segunda historia, un verdadero sentido para el deporte. Esta relación de sentidos y significados establece un sujeto, el atleta, como la expresión concreta de su forma de existencia en el mundo, en su grupo social, en el deporte donde se encuentra la expresión de su identidad, que es dinámica, envolviendo las circunstancias cotidianas y las relaciones entre similares y diferentes, así como presente, pasado y futuro (13).

Los atletas se destacan por la calidad técnica y la identificación con el público, convirtiéndose en un ídolo, héroe invencible y puede ser visto en forma mítica, por lo tanto, inmortal y el atleta, puede convertirse en su identidad, haciendo que simbólicamente se sienta capaz de dominar cualquier obstáculo, incluyendo la muerte, incluso teniendo problemas del corazón (12).

El jugador que actúa en un contexto de significados y emociones, con la intención, de que puede dar sentido a su vida. Pensando en sí mismo como enfermo es tener que replantear su propio significado. El cuerpo le da la conciencia de la individualidad, donde se establece la frontera entre la salud y la enfermedad, es también a través del cuerpo que pueda producirse el proceso de identificación con los demás (13). Cuando el atleta sano se identifica con el atleta con enfermedades del corazón, el pensamiento puede dictar que el mismo puede tener esta enfermedad, y ya no pueden desempeñar su papel, pueden experimentarse identidades perdidas, falta de reconocimiento social, y ya no existir más como un deportista.

Es para aquellos responsables del deporte, la relación de diálogo y dialéctica con la discusión de la salud y la enfermedad, escuchando al propio atleta, sujeto de la acción y por lo tanto entender.

El objetivo principal de este estudio es el aspecto subjetivo se refiere al atleta, que es uno de los temas de importancia para reducir al mínimo el riesgo de muerte inminente que es importante para el atleta contemporáneo, ya que es él, el principal actor del evento cardiaco.

\section{MATERIAL Y METODOS}

Entender el significado y la importancia del riesgo para desafiar el límite entre la vida y la muerte 
MAYNARD S.K. \& VARGAS A.L.S. Muerte súbita en los atletas: la frontera entre la vida y la muerte Mot. Hum., 11(2): 52-58, 2010.

durante la enfermedad del corazón de un atleta se expresa en los discursos de los atletas entrevistados. Analizar el discurso de los atletas, a quienes pertenece la responsabilidad de cesar la práctica deportiva debido al riesgo de muerte súbita por enfermedad cardíaca.

Investigación de análisis fenomenológico de los discursos.

Dieciséis deportistas de alto nivel en las modalidades de fútbol profesional, fútbol de sala y la lucha libre como MMA, con domicilio en Río de Janeiro, de sexo masculino, con edades comprendidas entre $17 \mathrm{y}$ 30 años, participando en competiciones deportivas de alto rendimiento.

Primera Entrevista Pregunta: ¿Díganos lo que piensa sobre el atleta que tiene que tomar la decisión de alejarse del deporte debido a tener enfermedades del corazón? Debía saber cuál es la idea que tiene en la toma de decisiones antes del hecho, la segunda pregunta: $\mathrm{Si}$ el doctor dice ahora que tiene una enfermedad cardiaca grave y debe retirarse definitivamente de la práctica deportiva, ¿cuál sería su reacción? Para identificar el sentido del riesgo de permanecer en el deporte a pesar de la enfermedad, la tercera pregunta: Si el examen del corazón mostrase problemas cardiacos, ¿usted desafiaría la muerte para continuar en el deporte? ¿Por qué? para identificar su disposición a asumir riesgos de muerte para el deporte, la cuarta pregunta: ¿Quién crees que debería ser responsable de la decisión de cesar la práctica deportiva? ¿El propio atleta, entrenador, médico, gerente del club o alguien más? identificar la idea de que el atleta tiene la responsabilidad profesional de cesar la actividad de él mismo, debido a las enfermedades del corazón.

\section{RESULTADOS}

La primera pregunta identificó que para este grupo de atletas, la situación es muy delicada y embarazosa expresadas por las palabras "... muy difícil ...", donde" muy "sugiere que la intensidad de la situación y"...difícil ... "la ambigüedad de la situación. Las palabras "... tener que parar ..." reflejan el sentimiento que existe de obligación de parar la práctica deportiva. En la toma de decisiones, hay una posición, que muestra el conflicto de ideas, y la indecisión, relacionadas con la extracción y el cese obligatorio.

Este análisis coincide con Epiphanio (9) relativo a los conflictos de la toma de decisiones y la angustia de asumir las consecuencias de la elección, debido a la importancia del deporte en sus vidas. Para un pequeño número de atletas, la toma de decisiones se expresa como un requisito.

La segunda cuestión se encontró que la reacción de los deportistas se basa en la emoción, que se dividió entre la decisión de continuar y/o detenerse mostrando indecisión ante el hecho. La decisión de continuar, expresada en las palabras "oh, no sé, estamos allí para morir de todos modos," hasta "yo mismo entraría en depresión, no pararía de entrenar, moriría entrenado"," ... no tendría nada en cuenta, seguiría haciendo deporte incluso si estoy mal "y" no podría soportar, juego para vivir "refleja el desprecio por la vida, en detrimento de otra situación que es más importante para él, en un proceso de desvalorización de sí mismo. La decisión de parar, relatado en las palabras: "Yo me detendría de inmediato!", "Yo paro en ese instante "Refleja que la vida es un bien mayor que cualquier otro, como el derecho a la vida con responsabilidad. La indecisión en las siguiente conversaciones "muy nervioso... no sé ..."," pánico" y "... lloraría mucho, estaría decepcionado .." podrían demostrar la incapacidad para la toma de decisiones.

El uso del verbo "parar" como reacción, se refiere a la interrupción de sus actividades deportivas y "no parar" es una negación de esta interrupción. La reacción de estos atletas demuestra una emoción que impulsa el sentido de la permanencia en el deporte (12).

En la tercera cuestión, el grupo de programas de los atletas que desafían la muerte, representa una pérdida para sí mismo, la vida, interpretada en las palabras "no, mi vida es más importante", que se repitieron durante la entrevista, en varias respuestas. El derecho a la vida por elección es evidente en este grupo, siendo ésta la primera razón para no enfrentar la muerte, y en seguida el valor relativo de la familia. Los que desafían a la muerte justifican su respuesta con la devaluación de su propia vida, los sentimientos de placer, la adicción y la posibilidad de lucro, identificada en los siguientes discursos "no 
MAYNARD S.K. \& VARGAS A.L.S. Muerte súbita en los atletas: la frontera entre la vida y la muerte Mot. Hum., 11(2): 52-58, 2010.

pararía no, está en la sangre, es una adicción es una adicción", " si lo enfrentaría por una apoyo económico (dinero) importante ", "con certeza, nada se compara a hacer lo que uno gusta, me da mucho placer ", sin darse cuenta de que la muerte es el fin material.

La cuarta y última pregunta, puso de manifiesto que a pesar del proceso tecnológico de la profesionalización del deporte, el atleta se somete, a la responsabilidad de cesar la actividad por contar con enfermedades del corazón, casi por unanimidad en lo que se refiere el propio atleta, a menudo se repite la palabra discursos.

\section{CONCLUSIÓN}

Entender los dilemas, escuchar a la persona del deportista, ofreciéndole opciones, orientándolos para mantenerse financieramente y no sólo a suspender sus actividades, es una actitud de valor en relación con el atleta. Al descubrir un portador de enfermedades del corazón no siente la identidad y no siempre consigue discernir la gravedad de la situación, siendo la responsabilidad profesional el valor que se refleja en la toma de la decisión, no solo para su seguridad física sino también para la emocional.

El estudio concluyó que las dificultades con la toma de decisiones, las emociones con respecto a la posibilidad de presentar enfermedades del corazón, identificadas en esta investigación, no impidió a los atletas de hablar sobre el significado de su vida y después, el sentido de la familia de su existencia, y el reto a la muerte representa la pérdida de sí mismo.

Paradójicamente, estos atletas no se sienten excluidos como personas, el proceso de profesionalización, que se atribuyen a sí mismos, tener la responsabilidad de la decisión de cesar sus actividades, debido a las enfermedades del corazón, expresando que la vida les pertenece a ellos y sobre ella se toman las decisiones, aun con el riesgo de muerte súbita.

Sobre los responsables de las actividades deportivas cae la relación dialógica y dialéctica con una discusión del proceso de salud y enfermedad, para conocer y entender al atleta, antes de que el significado y el propósito que construye su historia, que es la expresión concreta de su existencia en el mundo, y en su grupo social: el deporte. Por lo tanto, los casos de muerte súbita, como Serginho, y otros atletas han sucumbido ante los ojos de sus fans en tiempo real, y podrán ser evitadas.

\section{REFERENCIAS BIBLIOGRAFICAS}

1. Stein R. La muerte súbita relacionadas con el ejercicio: la actividad física, desde varios ángulos [sitio en Internet] 2005 [consultado en septiembre de 2008]. Disponible en: http://www.drashirleydecampos.com.br/noticias/1504 8.

2. Oliveira CM. Responsabilidad en el deporte. Análisis jurídico de la responsabilidad civil y penal en la reciente muerte del futbolista Serginho de Sao Caetano. Jus Vigilantibus [serie en Internet] 2004 [citado julio de 2008]. Disponible en: <http://jusvi.com/doutrinas_e_pecas/ver/2582>.

3. Cerqueira TTPL, Gomes LF. La trágica muerte del jugador Serginho e imputación objetiva. Rev Síntesis de Derecho Penal y Procesal Penal [serie en Internet] diez / enero 2005 [consultado en diez de 2008], 5 (29). Disponible en: www.mp.rn.gov.br/bibliotecapgj/sumarios/sumario_0 4-2005.pdf.

4. Oliveira MAB. La miocardiopatía hipertrófica, la actividad física y la muerte súbita. Rev Bras Med Esporte. 2002: 8 (1): 20-5.

5. Dippe Jr. T. Podemos predecir la muerte súbita? [Ubicado en Internet] 2008 [citado noviembre de 2007]. Disponible en: www.portaldocoracao.com.br/dr-tufi-dippejr.php?id=1357>.

6. Ghorayeb N, Batlouni M, Dioguardi GS, Timerman S, Nakhlawi A, Tricot PM, Dall Orto CC; Mugrabi DF. Muerte súbita cardíaca en deportistas: paradoja de la prevención posible. Rev El corazón, el deporte y ejercicio II. São Paulo: Soc. Cardiol, 2005: 15 (3); 242 -5.

7. Javornick R. Muerte súbita en deportistas: el papel del entrenador lugar [en Internet] 2001 [citado agosto de 2008] Disponible en: 
MAYNARD S.K. \& VARGAS A.L.S. Muerte súbita en los atletas: la frontera entre la vida y la muerte Mot. Hum., 11(2): 52-58, 2010.

http://www.gssi.com.br/scripts/publicacoes/treinador /treinador.asp

8. Maron BJ, Hacedor JJ, Haas TS, Tierney MD, Mueller. C Sudden deaths in young competitive athletes: analysis of 1866 deaths in the United States, 1980-2006.Circulación 2009: 120 (16);143144.

9. Ceunick MD, D'Hooghe M, D'Hooghe P. La muerte cardiaca súbita en el fútbol. Fútbol Manual de Medicina. $2005 \quad$ Capítulo 4.5.

10. Epiphanio EH. Los conflictos experimentados por los deportistas y el mantenimiento de los deportes de alto rendimiento. Rev. Estudios de Psicología 2002: 19

(1);

15-22.

11. Vaz FA. Teoría Crítica del Deporte: orígenes, polémicas y actuales. Revista Digital de Deporte y Sociedad.2007: 3 (7).

12. VLM Costa. Deportes de Aventura y Riesgo en la montaña: Un viaje a los límites del juego y la incertidumbre. Río de Janeiro (RJ): Tesis de Doctorado en Educación Física de la Universidad Gama $\quad$ Filho, 1999.

13. Koliniak Hijo C; Koliniak HMR. El deporte es salud? PUCVIVA Magazine [serie en Internet] 2005 [consultado en septiembre de 2009] 22. Disponible en:

http://www.apropucsp.org.br/revista/r22_r06.htm. 9.

14. Viana E. El poder en el deporte. Río de Janeiro (RJ): Sprint, 1994.

15. Marques AAC. Muerte súbita en la actividad física. Diario de la Educación Física [serie en Internet] 1993 [citado agosto de 2009] 121. Disponible en: http://www.revistadeeducacaofisica.com.br/paginas/ arevista.htm.

16. La Mendola S. O sentido do risco. Tempo Social, revista de sociologia da USP 2005: 17, (2); 59-91

17. Rocha VM. B.a.s.e. jump, risco e emocao: umaexperiencia para dar sentido a vida. Esporte e Sociedade 2008:
18. Raveneau G. Dossier: Sports à risques, corps du risque. Ethnologie Française, núm. 4. París

19. Kretly V, Oliveira EM, Breton ACP. La importancia del deporte para el atleta. Un estudio con los atletas del Centro Olímpico de Entrenamiento e Investigación. Acta Enf Pablo. 2003, $\quad 16 \quad$ (1): $66-75$.

20. H. Beresford H. Valor: sabe lo que es. Río de Janeiro (RJ): Forma, 2000

21. Manuel Sergio. Para una epistemología de Movimiento Humano. Lisboa (PT): Compendio de 1994. 
MAYNARD S.K. \& VARGAS A.L.S. Muerte súbita en los atletas: la frontera entre la vida y la muerte Mot. Hum., 11(2): 52-58, 2010.

\begin{abstract}
To understand the meaning and significance of risk in challenging the boundary between life and death during an athlete's heart disease, expressed in the speeches of the interviewees, under the question: who owns the responsibility of the athlete's removal at risk for heart disease? Methodology phenomenological research of the type of discourse analysis. The decision has generated conflict, the anguish of assuming the consequences of choice, by the significance of sport in their lives. The emotion was the basis of the reaction. There was division between the decision to continue, stop and indecision on facing the fact. They grant to themselves the right to choose. The decision of removal is taken by the athlete himself, expressing that life belongs to them and has the sense of their existence. They make the decisions about it, showing the significance of the sport, even at the risk of death.
\end{abstract}

Key words: risk, cardiac sudden death, death

Dirigir correspondencia a:

Maynard da Silva, Kenia

End: Av. das Américas 1245 apto 2202/Rio de Janeiro/RJ/

BRASIL

CEP:22631-000

Email: kenia.msilva@gmail.com

RECIBIDO 8-12-2010

ACEPTADO 20-12-2010 